\title{
A Method of Skipping the Transient Phase in the Solution of Separably Stiff Ordinary Initial Value Problems
}

\author{
By Peter Alfeld
}

\begin{abstract}
Stiff systems of ordinary differential equations are characterized by an initial phase in which the solution changes rapidly. Often there is no interest in reproducing this transient phase. A method is proposed for modifying the initial value if the system of differential equations is separably stiff, i.e. is characterized by the occurrence of a few (typically one) large negative real eigenvalues which dominate the others. The modified system does not possess a transient phase, and in the constant coefficient linear case its solution does not differ from that of the original one in the nonstiff components.
\end{abstract}

1. Introduction. In order to avoid repetition, the reader is assumed to be familiar with reference [1], particularly with Sections 1, 2, 3-III, and 5-III. We will use the notation given there and consider separably stiff initial value problems (IVPs).

$$
y^{\prime}=f(x, y), \quad y(a)=\eta, \quad y, f \in \mathbf{R}^{m} .
$$

For convenience, we restrict ourselves to the case $s=1$. In this note, we point out that the Gradient Projection CDS technique can be applied to the initial value $\eta$ in (1.1). The result is a modified IVP whose solution, in the constant coefficient linear case, differs from that of (1.1) only in the stiff component. In the variable coefficient and in the nonlinear case, the solution of the modified problem is an approximation to the solution of the original problem. In both cases, the solution of the modified problem does not possess a transient phase.

2. The Linear Case. We first consider the constant coefficient linear problem

$$
y^{\prime}=A y+g(x), \quad y, g \in \mathbf{R}^{m}, \quad y(a)=\eta, \quad x \in[a, b],
$$

where $A$ is a constant separably stiff nondefective matrix. The solution of (2.1) can be written as

$$
y(x)=\sum_{i=1}^{m} \phi^{(i)}(x) c^{(i)}, \quad \phi^{(i)}(x)=\left\langle d^{(i)}, y(x)\right\rangle,
$$

and its derivative as

$$
y^{\prime}(x)=\sum_{i=1}^{m} \psi^{(i)}(x) c^{(i)}, \quad \psi^{(i)}(x)=\left\langle d^{(i)}, y^{\prime}(x)\right\rangle
$$

Received October 18, 1979.

1980 Mathematics Subject Classification. Primary 65L05.

Key words and phrases. Stiff ordinary differential equations, numerical analysis, separably stiff systems, transient phase. 
(Here $\psi^{(i)}=\left(\phi^{(i)}\right)^{\prime}$, but this will not be true in the generalization to the variable coefficient and the nonlinear case.)

Since $A$ is separably stiff (with $s=1$ ), the only stiff component of $y(x)$ is $\phi^{(1)}(x)$. This suggests modifying the initial value $\hat{\eta}=\eta+\xi c^{(1)}$, such that $\left\langle d^{(1)}, f(a, \hat{\eta})\right\rangle=0$, and then solving the IVP

$$
y^{\prime}=A y+g(x), \quad y, g \in \mathbf{R}^{m}, \quad y(a)=\hat{\eta}, \quad x \in[a, b] .
$$

The exact solution of (2.2) is given by

$$
\hat{y}(x)=\sum_{i=1}^{m} \hat{\phi}^{(i)}(x) c^{(i)},
$$

and its derivative by

$$
\hat{y}^{\prime}(x)=\sum_{i=1}^{m} \hat{\psi}^{(i)}(x) c^{(i)}
$$

where

$$
\phi^{(i)}=\hat{\phi}^{(i)} \text { and } \quad \psi^{(i)}=\hat{\psi}^{(i)} \text { for } 2 \leqslant i \leqslant n \text {, }
$$

and

$$
\hat{\psi}^{(1)}(a)=0
$$

The relation (2.4) ensures that $\hat{y}$ does not possess a transient phase.

In the variable coefficient case,

$$
y^{\prime}=A(x) y+g(x), \quad y(a)=\eta,
$$

we replace $\eta$ by

$$
\hat{\eta}=\eta+\xi c^{(1)}(a)
$$

and choose $\xi$ so as to satisfy

$$
\left\langle d^{(1)}(a), f(a, \hat{\eta})\right\rangle=0
$$

Due to interprojection effects (see [2] for a detailed description) the relations (2.3) are now no longer satisfied, i.e. the modification of the initial value affects all components of $\hat{y}(x)$. However, (2.4) is still true, and hence $\hat{y}(x)$ does not possess a transient phase.

The correction factor $\xi$ in (2.5) easily computes to

$$
\xi=-\left\langle d^{(1)}(a), f(a, \eta)\right\rangle / \lambda^{(1)}(a)
$$

see [1, Section 3-III]

3. The Nonlinear Case. Consider the fully nonlinear IVP (1.1). The dominant eigensystem now depends on the initial value. In [1, Section $5-\mathrm{III}]$, it is assumed that any corrections are so small that the dominant eigensystem at the uncorrected value can be used for the gradient projection. Since we contemplate a potentially large modification of the initial value, this is clearly inadequate in the present context. 
Therefore, we replace $(2.5)$ and $(2.6)$ by

$$
\hat{\eta}=\eta+\xi c^{(1)}(a, \hat{\eta})
$$

and

$$
\left\langle d^{(1)}(a, \hat{\eta}), f(a, \hat{\eta})\right\rangle=0,
$$

respectively. The resulting implicitness can be tackled by using the iteration

$$
\left\{\begin{array}{l}
\xi^{[0]}=0, \quad \eta^{[0]}=\eta \\
\text { For } t=0,1,2, \ldots \\
\eta^{[t+1]}=\eta^{[t]}+\xi^{[t]} c^{(1)}\left(a, \eta^{[t]}\right) \\
\xi^{[t+1]}=\xi^{[t]}-\frac{\left\langle d^{(1)}\left(a, \eta^{[t]}\right), f\left(a, \eta^{[t]}\right)\right\rangle}{\lambda^{(1)}\left(a, \eta^{[t]}\right)}
\end{array}\right.
$$

which is a natural generalization of the iteration (5.2) in [1]. Note that, for a separably stiff system, the updates of the dominant eigensystem required in (3.1) can be carried out very efficiently by the power method.

4. Numerical Example. We consider the following severely stiff naturally arising problem given in [3].

$$
\begin{array}{ll}
y_{1}^{\prime}=-y_{1}+10^{8} y_{3}\left(1-y_{1}\right), & y_{1}(0)=1, \\
y_{2}^{\prime}=-10 y_{2}+3 \cdot 10^{7} y_{3}\left(1-y_{2}\right), & y_{2}(0)=0, \quad x \in[0,1] . \\
y_{3}^{\prime}=-y_{1}^{\prime}-y_{2}^{\prime}, & y_{3}(0)=0 .
\end{array}
$$

The modified initial value, obtained by the iteration ( 3.1$)$, is

$$
\begin{aligned}
& \eta_{1}=9.72034445009242292 \mathrm{D}-1, \\
& \eta_{2}=2.79655158235541304 \mathrm{D}-2 \\
& \eta_{3}=3.91672035777926715 \mathrm{D}-8
\end{aligned}
$$

A variable-step-variable-order integration package based on blended linear multistep methods [4] was applied to this problem with different error tolerances. The initial step-size given to the routine was $h=0.1$, which is much too large; the idea being that the algorithm will decrease this step-length to an acceptable size.

The following table contains:

$\epsilon$ : error tolerance requested,

$h_{0}$ : initial step-size chosen by the algorithm for the unmodified problem,

$\hat{h}_{0}$ : initial step-size chosen by the algorithm for the modified problem,

$\Delta: \max _{k=1,2, \ldots, 10}\|y(0.1 k)-\hat{y}(0.1 k)\|_{\infty}$, where $y$ and $\hat{y}$ were computed at the grid-points by interpolation using the scaled derivative estimates given by the algorithm. 


$$
\begin{array}{cccc}
\multicolumn{4}{c}{\text { Table of numerical results }} \\
\epsilon & h_{0} & \hat{h}_{0} & \Delta \\
10^{-3} & 1.24 \mathrm{D}-3 & 1.45 \mathrm{D}-2 & 1.73 \mathrm{D}-5 \\
10^{-4} & 1.39 \mathrm{D}-4 & 4.58 \mathrm{D}-3 & 7.55 \mathrm{D}-6 \\
10^{-5} & 1.22 \mathrm{D}-5 & 1.44 \mathrm{D}-3 & 7.83 \mathrm{D}-6 \\
10^{-6} & 1.24 \mathrm{D}-6 & 4.56 \mathrm{D}-4 & 7.62 \mathrm{D}-6 \\
10^{-7} & 1.07 \mathrm{D}-7 & 1.44 \mathrm{D}-4 & 7.65 \mathrm{D}-6
\end{array}
$$

We see that the initial step-size is significantly larger for the modified problem, indicating that we effectively skipped the transient phase. The values for $\Delta$ suggest that the exact solutions differ globally beyond the transient phase $(0.1$ is well outside the transient phase) by less than $10^{-5}$.

Conclusions. A technique has been described that allows for separably stiff systems effectively to skip the transient phase if there is no interest in reproducing it. Apart from possibly increasing the efficiency of stiff O.D.E. solvers, this technique can also be used to find initial conditions that are of physical interest for the reason that they do not create a transient phase.

Department of Mathematics

University of Utah

Salt Lake City, Utah 84112

1. P. ALFELD \& J. D. LAMBERT, "Correction in the dominant space: A numerical technique for a certain class of stiff initial value problems," Math. Comp., v. 31, 1977, pp. 922-938.

2. P. ALFELD, "A special class of explicit linear multistep methods as basic methods for the correction in the dominant space technique," Math. Comp., v. 33, 1979, pp. 1195-1212.

3. W. H. ENRIGHT, T. E. HULL \& B. LINDBERG, "Comparing numerical methods for stiff systems of ODEs," BIT, v. 15, 1975, pp. 10-48.

4. R. D. SKEEL \& A. K. KONG, Blended Linear Multistep Methods, Report UIUCDCS-R76-800, Department of Computer Science, University of Illinois at Urbana-Champaign, 1976. 